3. Smith R (2008) Why do we need Cases Journal? Cases J 1(1):1

4. Celebrating case reports, the stories in health care - videos from Cases Network. (2009) http://www. casesnetwork.com/symposium/Jefferson.html

5. Aronson JK, Hauben M (2006) Anecdotes that provide definitive evidence. BMJ 333(7581):12671269

6. Glasziou P, Chalmers I, Rawlins M, McCulloch P (2007) When are randomised trials unnecessary? Picking signal from noise. BMJ 334(7589):349-351

7. Loke YK, Price D, Derry S, Aronson JK (2006) Case reports of suspected adverse drug reactions systematic literature survey of follow-up. BMJ 332(7537):335-339

8. Richason TP, Paulson SM, Lowenstein SR, Heard KJ (2009) Case reports describing treatments in the emergency medicine literature: missing and misleading information. BMC Emerg Med 9:10

Intensivmed 2009 · 46:375

DOI 10.1007/s00390-009-0109-1

Online publiziert: 3. September 2009

(c) Springer-Verlag 2009

\title{
S. Kluge
}

Klinik für Intensivmedizin, Universitätsklinikum Hamburg-Eppendorf, Hamburg

Für Sie gelesen

\section{Die Rubrik,Journal Club"}

In der Rubrik „Journal Club“ sollen in jeder Ausgabe intensiv- und notfallmedizinisch relevante Publikationen aus der internationalen Fachliteratur von Experten für Sie bewertet und kommentiert werden.

Für Intensiv- und Notfallmediziner wird es immer schwerer, sich bei der wachsenden Anzahl von Veröffentlichungen einen umfassenden Überblick zu verschaffen. Die Rubrik „Journal Club“ soll Ihnen hierbei helfen und insbesondere für Sie praxisrelevante Studien identifizieren und kommentieren. Den Lesern soll dadurch die unkomplizierte Umsetzung neuester Forschungsergebnisse in ihrer täglichen Praxis erleichtert werden. Um den Einstieg in die kommentierte Studie zu vereinfachen, wird in jedem Beitrag zunächst die Originalpublikation zusammengefasst. Dadurch wird ein Überblick über die Originaldaten gewährleistet.

Im anschließenden Kommentar reflektiert ein klinisch und wissenschaftlich qualifizierter Experte die vorgestellten Daten, insbesondere unter dem Aspekt der evidenzbasierten Medizin, und leitet daraus ein „Fazit für die Praxis“ab.

Wir hoffen, dass „Intensivmedizin und Notfallmedizin“ Ihnen mit der Rubrik „Journal Club“ einen kompetenten Überblick über die internationale Fachliteratur gibt. Die Kommentare sollen es Ihnen ermöglichen, eine kritische Einschätzung der Publikationen vorzunehmen und sich selbst eine differenzierte Meinung zu bilden.

Ihr

Dr. Stefan Kluge

\section{Korrespondenzadresse}

Dr. S. Kluge

Klinik für Intensivmedizin, Universitätsklinikum Hamburg-Eppendorf

Martinistr. 52, 20246 Hamburg

skluge@uke.de 